\title{
UNUSUAL FOREIGN BODY [TOOTH BRUSH] IN THE MASSETER MUSCLE A RARE CASE REPORT
}

\author{
Krushna Chandra Mallik, Ranjan Kumar Guru
}

1. Associate Professor, Department of ENT, VSS Medical College, Burla, Sambalpur,Odisha.

2. Senior Resident, Department of ENT, VSS Medical College, Burla, Sambalpur,Odisha.

\author{
CORRESPONDING AUTHOR: \\ Ranjan Kumar Guru, \\ Senior Resident, \\ Department of ENT, \\ VSS Medical College, burla \\ Sambalpur, Odisha, pin - 768017. \\ E-mail: dsnh@rediffmail.com
}

\begin{abstract}
OBJECTIVE : - This case is reported for emphasizing on early diagnosis in suspicious cases of foreign body lodgement in any age group by detail history taking and relevant radiography, thereby managing early \& successfully. Early removal of foreign body will prevent further complications. STUDY DESIGN: - Retrospective study. SETTING: - VSS Medical College \& Hospital, Burla, Sambalpur, Odisha. PATIENT: A 62 year aged male with suspicious osteomyelitis of lower jaw which after CT scanning revealed a lodgement of foreign body (Tooth Brush) in the right cheek. RESULT: - By performing CT scan the foreign body Tooth Brush was diagnosed in the right masseter muscle. Tooth brush was removed under general anaesthesia by transverse incision over right cheek. CONCLUSION: - A wide range of suspicion of clinician, during and after taking history by the clinician definitely helps in early diagnosis \& early intervention to prevent complications. Educating the patient is also equally important.
\end{abstract}

KEY WORDS: - Foreign body, Tooth brush, Masseter muscle.

INTRODUCTION: - A foreign body (Latin: corpus alienum) is any object originating outside the body. In machinery, it can mean any unwanted intruding object. Foreign bodies can be inert or irritating. If they irritate they will cause inflammation and scarring. They can bring infection into the body or acquire infectious agents and protect them from the body's immune defences. They can obstruct passageways either by their size or by the scarring they cause. Some can be toxic.

Both children and adults experience problems caused by foreign objects getting stuck in their bodies. Young children, in particular, are naturally curious and may intentionally put shiny objects, such as coins or button batteries, into their mouths. They also like to stick things in their ears and up their noses [1]. The effect of a foreign body can be very different. For example, a coin causes local pressure on the tissue but generally, is not a medical emergency to remove. A button battery, which can be a very similar size to a coin, generates hydroxide ions at the anode and causes a chemical burn in 2 hours [2]. An ingested button battery that is stuck in the esophagus is a medical emergency. In 2009, Avolio Luigi and Martucciello Giuseppe showed that although ingested nonmagnetic foreign bodies are likely to be passed spontaneously without consequence, ingested magnets (magnetic toys) may attract each other through childrens' intestinal walls and cause severe damage, such as pressure necrosis, perforation, intestinal fistulas, volvulus, and obstruction. [3]. 


\section{CASE REPORT}

Removal of the foreign bodies is essential to prevent super-infections, abscesses and perforations with potentially life threatening mediastinal complications in case of esophageal foreign bodies [4]. Although rarely, foreign bodies sometimes migrate within the tissues and become symptomatic after a certain time lapse [5]. In those cases, the direct relation between the suspected foreign body ingestion and the first symptoms is rarely established due to the latency and unusual clinical presentation [6, 7].

Our case is a unique case as the history was misleading \& the clinical presentation was unusual.

CASE REPORT :-A 62 year aged male admitted to the department of ENT \& Head \& Neck Surgery, VSS Medical college, Burla, with chief complaints of persistent pain \& swelling over right cheek since last 4 months, which was associated with decrease mouth opening \& pain during opening of mouth. This pain \& swelling was also associated with intermittent foul smelling discharge from an opening over the skin of right cheek since last 3 month. After 1 month of the first episode of pain he developed a discharging opening over the skin of right cheek. Since then he is suffering from pain, swelling \& intermittent discharge from the right cheek. Subsequently he developed another opening in front of it with a whitish hard material coming out from the opening.

On physical examination there was an opening over the skin of right cheek, a small hard whitish mass was protruding about $2 \mathrm{~mm}$ from the skin opening through another opening(fig.1) . There was surrounding induration \& trismus was present. A hard concretion resembling the exposed bone of the lower jaw was there inside the gingivo labial sulcus on examination of oral cavity.(fig-2).

Basing on the history \& physical findings, we suspected provisionally a case of osteomyelitis of lower jaw. Keeping this in mind we advised a CT scan. The CT scan revealed that a foreign body resembling the bristle end of a tooth brush was lodged in the right masseter muscle \& it was extending into soft tissue of right cheek(fig.3). After careful \& detailed history taking, patient could remember and revealed that, 4 months back on one morning the person was brushing his teeth, at that time he suffered from an episode of head reeling \& fell down. He lost his consciousness for a transient period. When he became conscious he found that his tooth brush was broken, as only the handle of the tooth brush was in his hand \& the distal part containing the bristles was missing. When he searched for the broken part he could not got it \& he thought that the part of tooth brush containing the bristles was lost. During this episode he suffered an injury inside the buccal surface of his right cheek, there was no injury over the external surface of the cheek. For this injury he took some local medication \& got some temporary relief. Finally the diagnosis was established to a foreign body lodged in the masseteric region.

We explored the cheek under general anaesthesia by transverse incision over the cheek. To our utter surprise we discovered the bristle end of the tooth brush lodging in the right masseter muscle \& the other end was emerging out through the external wound over the cheek (fig. 4). The tooth brush was removed (fig. 5). The mandible was not at all involved. After removing the foreign body hemostasis was maintained \& wound stitched in layers(fig.6). The post operative period was uneventful.

DISCUSSION: - Foreign bodies impacted within the ENT sphere, typically fish bones, have been reported to cause upper respiratory airway tract abscesses [8]. However, the migration through the 
entire pharyngeal wall ending in a superficial cervical abscess several months later is uncommon but has to be considered $[9,10]$. Repeated abscesses which seem resistant to treatment should always evoke the possibility of a foreign body or an underlying congenital malformation such as branchial cleft cysts [8], even if radiological examination fails to evidence its presence.

A majority of ingested FB pass through the gastrointestinal tract uneventfully. Severe complications are rare and often associated with delayed discovery due to silent and protracted clinical manifestations such as new onset asthma, excessive salivation or recurrent upper respiratory infections [5]. These undetected FB tend to create fistulas to the surrounding structures (e.g. aorta, bronchus, etc.) leading to potential life-threatening situations [5]. In contrast to adults, where symptoms and information on the swallowed object facilitates the diagnostic and therapeutic approach, children often present with few or absent symptoms and absence of symptoms does not preclude the presence of a FB [11]. However the detection of a foreign body and the follow-up of the clinical course is crucial, especially since complications even sometimes occur after it has been extracted [12].

While FB migration has been reported in adults $[9,13]$, the present case reports this rare complication in an old man. Particularly, the FB's nature seems uncommon, even amongst adult reports [9]. Even though a tooth brush is not sharp \& unusual to lodge in the cheek, depending on the force of impact it can break \& enter into the soft tissue of the cheek. In the present case the strong force of impaction over the tooth brush due to fall, might have facilitated the initial tissue penetration into the soft tissue of cheek \& masseter muscle.

CONCLUSION: - Hence in our view in the absence of positive history, early diagnosis by proper radiological investigations \& removal by expert with appropriate instruments are key to prevent complications in case of impacted foreign bodies anywhere in the body.

\section{ABBREVIATIONS:-}

CT - Computed tomography

FB - Foreign body.

\section{REFERENCES:-}

1. "Foreign Bodies: Nose and Paranasal Sinus Disorders: Merck Manual Professional". Retrieved 2008-12-16.

2. Litovitz, Toby. ."Swallowed a Button Battery?" National Capital Poison Center. Retrieved 11 June 2011.

3. Avolio L, Martucciello G.(2009 Jun)."Images in clinical medicine. Ingested magnets".N Engl J Med. 25; 360(26):2770.

4. Brinster CJ, Singhal S, Lee L, Marshall MB, Kaiser LR, Kucharczuk JC: Evolving options in the management of esophageal perforation. Ann Thorac Surg 2004, 77:1475-1483.

5. Gilchrist BF, Valerie EP, Nguyen M, Coren C, Klotz D, Ramenofsky ML: Pearls and perils in the management of prolonged, peculiar, penetrating esophageal foreign bodies in children. J Pediatr Surg 1997, 32:1429-1431.

6. Tsunoda K, Sakai Y, Watanabe T, Suzuki Y: Pseudo vocal paralysis caused by a fish bone. Lancet 2002, 360:907. 


\section{CASE REPORT}

7. Barzilai G, Braverman I, Karmeli R, Greenberg E: How did it get there? A coiled metal foreign body in an unusual cervical position. Otolaryngol Head Neck Surg 2001, 124:590-591.

8. Nusbaum AO, Som PM, Rothschild MA, Shugar JM: Recurrence of a deep neck infection: a clinical indication of an underlying congenital lesion. Arch Otolaryngol Head Neck Surg 1999, 125:1379-1382.

9. Gertner R, Bar'el E, Fradis M, Podoshin L: Unusual complication of an ingested foreign body. J Laryngol Otol 1991, 105:146-147.

10. Kumar BN, Walsh RM, Courteney-Harris RG: Laryngeal foreign body: an unusual complication of percutaneous tracheostomy. J Laryngol Otol 1997, 111:652-653.

11. Cheng W, Tam PK: Foreign-body ingestion in children: experience with 1,265 cases. J Pediatr Surg 1999, 34:1472-1476.

12. Wadie GM, Konefal SH, Dias MA, McLaughlin MR: Cervical spondylodiscitis from an ingested pin: a case report. J Pediatr Surg 2005, 40:593-596.

13. 13.Chee LW, Sethi DS: Diagnostic and therapeutic approach to migrating foreign bodies. Ann Otol Rhinol Laryngol 1999, 108:177-180.

\section{Figure - 1 foreign body lodged in cheek}

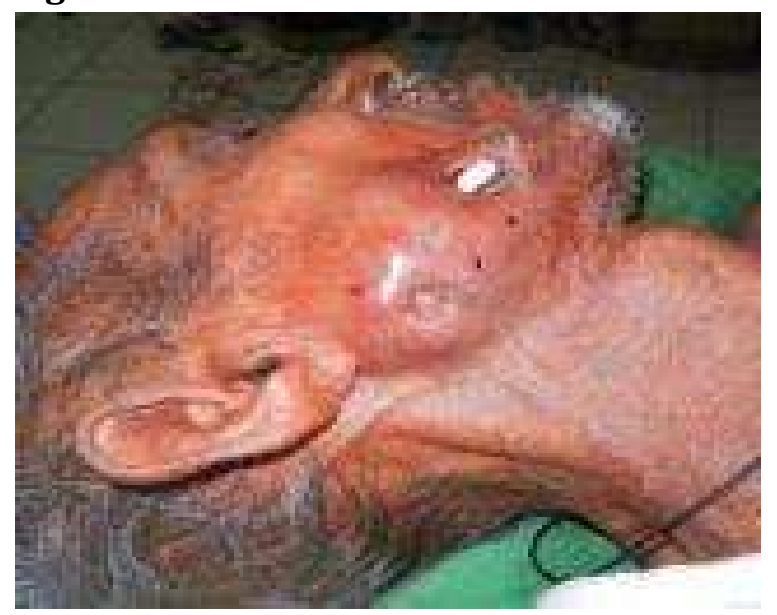

Figure -2 concretion in gingivolabial sulcus

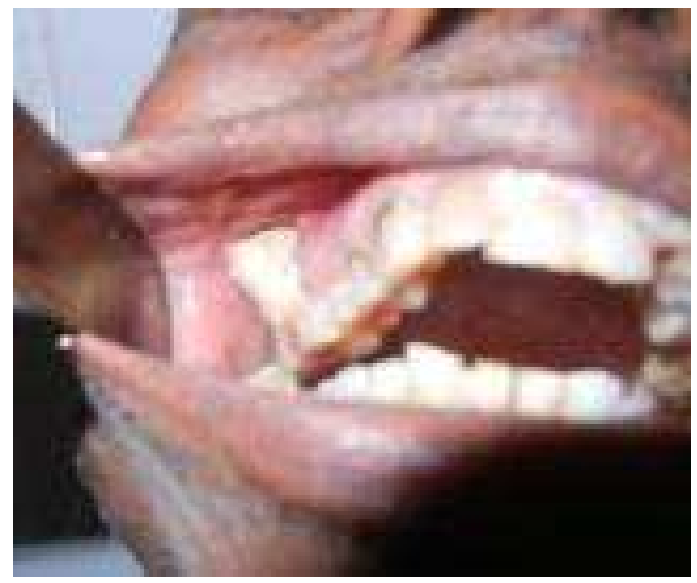




\section{CASE REPORT}

Figure - 3 ct scan showing tooth brush in masseter muscle

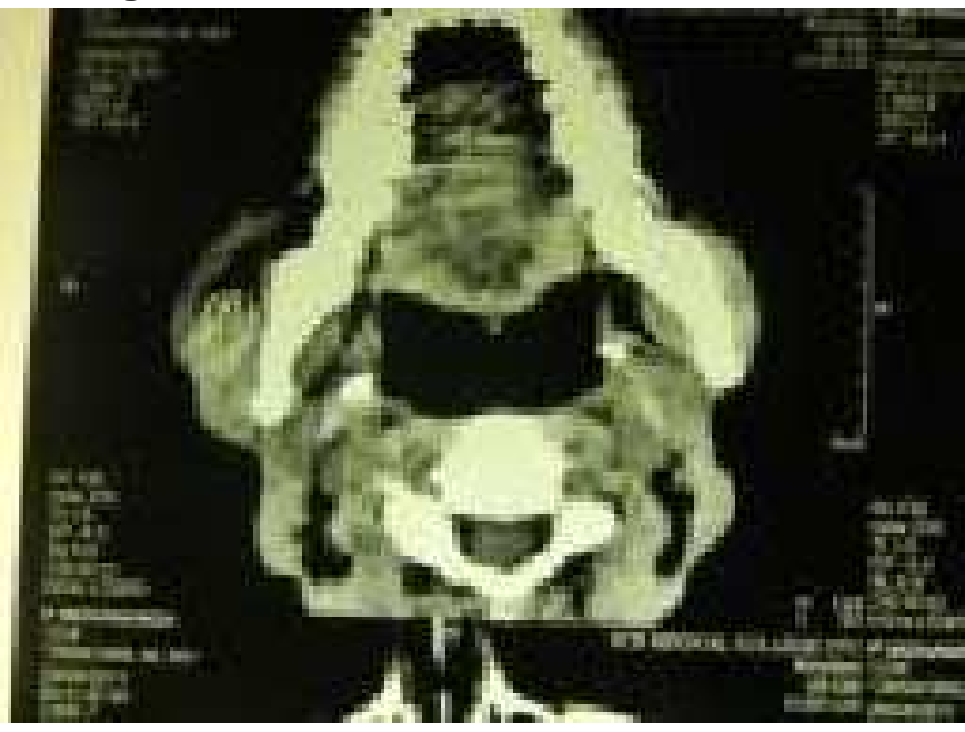

Figure -4 intraoperative picture

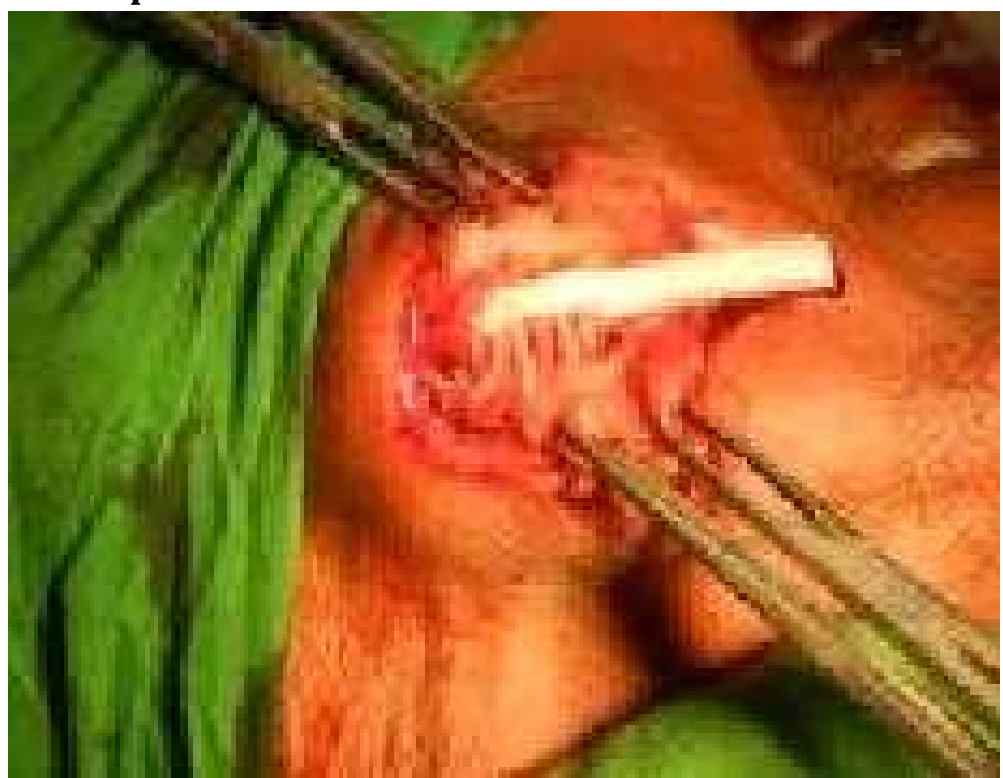




\section{CASE REPORT}

Figure - 5 tooth brush after removal

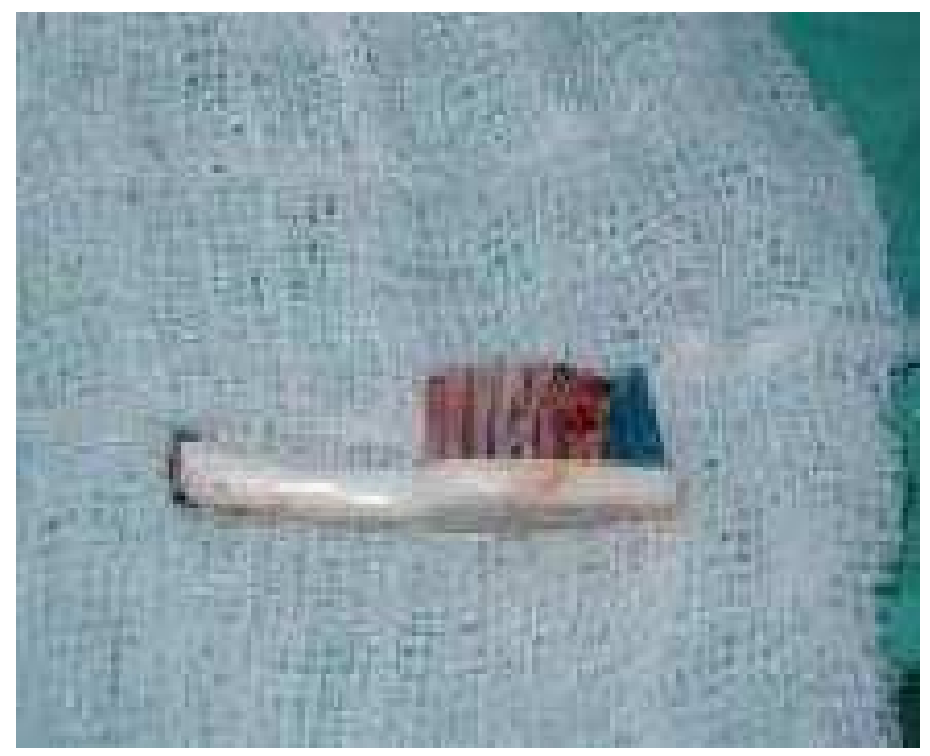

Figure -6 post operative picture

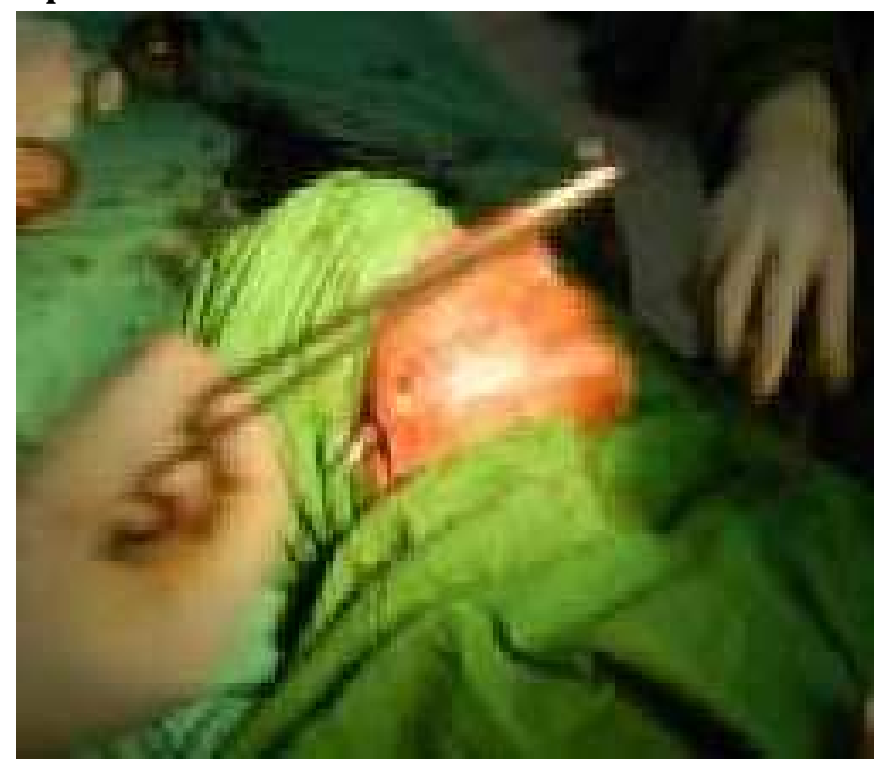

